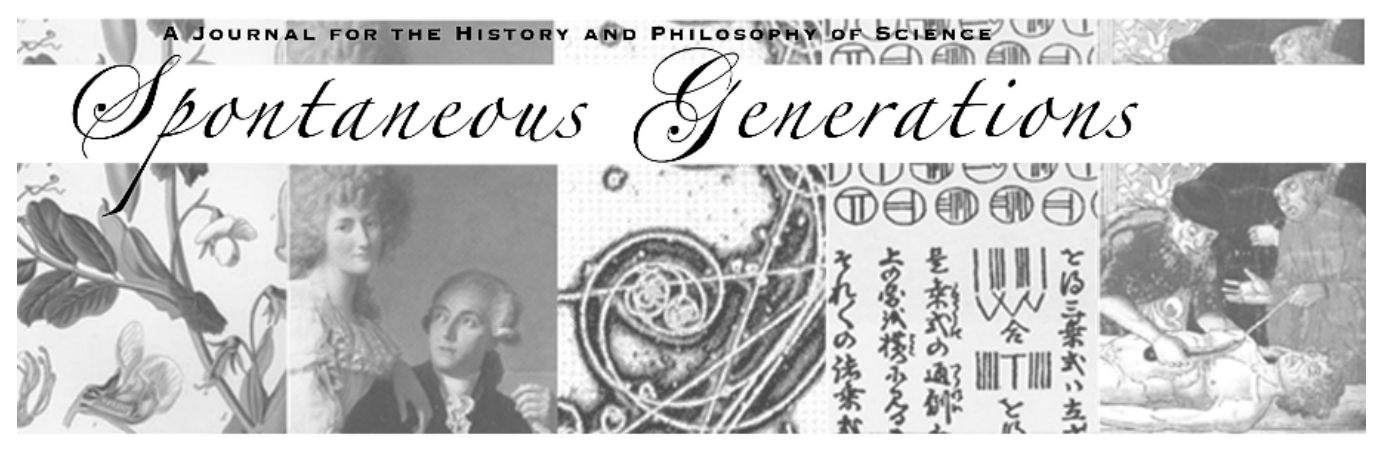

\title{
Interpreting Feynman Diagrams as Visual Models
}

\section{Author(s): Adrian Wüthrich}

Source: Spontaneous Generations: A Journal for the History and Philosophy of Science, Vol. 6, No. 1 (2012) 172-181.

Published by: The University of Toronto

DOI: 10.4245 /sponge.v6i1.16131

\section{EDITORIAL OFFICES}

Institute for the History and Philosophy of Science and Technology

Room 316 Victoria College, 91 Charles Street West

Toronto, Ontario, Canada M5S 1K7

hapsat.society@utoronto.ca

Published online at jps.library.utoronto.ca/index.php/SpontaneousGenerations ISSN 19130465

Founded in 2006, Spontaneous Generations is an online academic journal published by graduate students at the Institute for the History and Philosophy of Science and Technology, University of Toronto. There is no subscription or membership fee. Spontaneous Generations provides immediate open access to its content on the principle that making research freely available to the public supports a greater global exchange of knowledge. 


\title{
Interpreting Feynman Diagrams as Visual Models ${ }^{*} \dagger$
}

\author{
Adrian Wüthrich $\ddagger$
}

\begin{abstract}
I give a brief introduction to how Feynman diagrams are used. I review arguments to the effect that they are only used as calculation tools and should not be interpreted as representations of physical processes. Against these arguments, I propose to regard Feynman diagrams as visual models that explain, in some respects, how elementary particles interact.
\end{abstract}

\section{INTRODUCTION}

Anybody who opens a textbook on quantum field theory or has a look at a research publication on theoretical particle physics will encounter many more drawings than he or she would expect on the basis of the opinion that the essential content of theoretical physics is expressed in mathematical formulas. This view is expressed, more or less explicitly, in virtually any modern account of the application, interpretation and genesis of Feynman diagrams. They are viewed as mere calculation tools with mainly practical or pedagogical interest. James Robert Brown (1996, 265-67), for instance, says: "When Richard Feynman was working on quantum electrodynamics in the late 1940s, he created a set of diagrams to keep track of the monster calculations that were required. [...] We see the lines in the diagram; we do not visualize the physical process itself, nor any sort of abstract version of it." ${ }^{1}$

* Acknowledgments: Ari Gross as well as three anonymous referees have helped me enormously improve this article. Earlier drafts have been read and discussed by the group of visiting fellows in 2011 at the University of Pittsburgh's Center for Philosophy of Science and by Christian Wüthrich. Christoph Greub helped me with physical details and calculations. The Center for Philosophy of Science (Pittsburgh) funded the postdoctoral fellowship during which I began this article.

$\dagger$ Received 22 February 2012. Accepted 8 August 2012.

$\ddagger$ Adrian Wüthrich is a postdoctoral researcher, funded by the Swiss National Science Foundation, at the University of Bern, Switzerland. In 2009, he earned his PhD from the University of Bern in History and Philosophy of Science with a thesis on the genesis of Feynman diagrams. Apart from Feynman diagrams, his research is mainly concerned with principles of causality and locality in quantum mechanics and with the methodology and ontology or modern particle physics.

1 See also, among others, M.E. Peskin and D.V. Schroeder, An Introduction to Quantum Field Theory, (Boulder, CO: Westview Press, 1995, 5); S.S. Schweber, QED and the Men Who Made It:

Spontaneous Generations 6:1 (2012) ISSN 1913-0465. University of Toronto. 
Here (and on other occasions) I take issue with this view. For instance, my historical reconstruction of the development of Feynman diagrams (Wüthrich 2010) emphasizes that Richard P. Feynman used diagrams not only as calculation tools but also as theoretically motivated representations of physical processes. Moreover, the transition to Feynman diagrams, from representations which were in use before them, reflects significant changes in how quantum field theoretical phenomena were conceptualized and modeled (see Wüthrich 2010, section 1.8, and chap. 3). ${ }^{2}$

In the following, I will first briefly introduce the modern application of Feynman diagrams. Then I will present the two main objections to regarding Feynman diagrams as representations of real physical processes rather than as mere calculation tools. I will grant that these objections rightly dismiss certain types of interpretations, e.g. those which take the lines of a Feynman diagram to represent particle trajectories. However, I will also put forth arguments which can be found in the physics literature, that are based on Feynman diagrams, and, at least at first sight, cannot be dismissed by these objections. In conclusion, I will propose to regard Feynman diagrams as visual models which allow us to partially explain certain features of observed phenomena.

It remains to be seen whether or not, in the end, the aforementioned objections also bar the interpretation I propose. Still, I suggest that examples like the one I discuss below should be taken into serious consideration before one concludes that Feynman diagrams are only calculation tools.

While I will not give a precise definition of what constitutes a "representation," I should mention that my interpretation contrasts to, for example, Meynell (2008), as I conceive of (scientific) representations as always involving reference to a real physical process. In my view, a representation articulates some relevant aspects of a real physical system. Accordingly, the focus of the present paper is on whether we can regard Feynman diagrams as representations, understood along these lines, of real physical processes without running into contradictions with the principles of quantum mechanics.

\section{A CRASH COURSE in Feynman diagrams}

One of the most important quantities that characterizes a quantum field theoretical system is the probability of a transition from a given initial state to

Dyson, Feynman, Schwinger, and Tomonaga, (Princeton, NJ: Princeton University Press, 1994, 434); D. Griffiths, Introduction to Elementary Particle Physics, (Hoboken, NJ: Wiley, 1987, 59); and D. Kaiser, Drawing Theories Apart: The Dispersion of Feynman Diagrams in Postwar Physics, (Chicago: The University of Chicago Press, 2005, Chapter 1).

2 A few paragraphs of the present text are translated and revised pieces of one of my other publications. See A. Wüthrich, Zur Anwendung und Interpretation der Feynman-Diagramme. In Philosophie der Physik, ed. M. Esfeld, (Frankfurt a. M.: Suhrkamp, 2012; 227-44). 
a given final state. ${ }^{3}$ The initial and final states are often uniquely identified by the number and type of particles which are present in them and what momenta these particles have. Quantum field theory provides mathematical expressions which, when evaluated approximately, equal (approximately) the observed transition probabilities between initial and final states. No exact method of evaluating these expressions is known, and one necessarily has to resort to approximate methods. The most important of them, for the present purposes, is the so-called perturbative expansion. The main idea of the perturbative expansion is to replace expressions like $\sin (x)$ by an infinite series of additions or subtractions, i.e.

$$
\sin (x)=x-\frac{x^{3}}{3 \times 2}+\frac{x^{5}}{5 \times 4 \times 3 \times 2}-\frac{x^{7}}{7 \times 6 \times 5 \times 4 \times 3 \times 2}+\cdots
$$

and to consider only small values of $x$ such that higher powers of $x\left(\right.$ e.g. $\left.x^{3}\right)$ are much smaller than lower powers (e.g. $x$ ). Still, the more terms one includes in the series, the nearer the result gets to the theoretically defined value of $\sin (x)$. The equation $\sin (x)=x$ shows the approximation "to first order," which in this case is also the lowest, non-vanishing order. If one includes the subsequent terms, one is taking into account "corrections of higher order," which in this case are of third or higher order in the variable $x$.
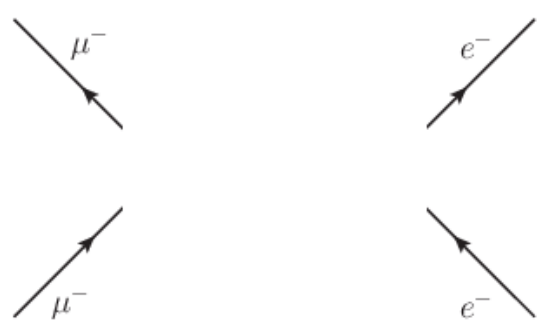

Figure 1. Adrian Wüthrich. First stage in the course of drawing a Feynman diagram used for the theoretical treatment of the mutual scattering of an electron $\left(\mathrm{e}^{-}\right)$and a muon $\left(\mu^{-}\right)$.

Instead of a mathematical derivation of the transition probabilities from the basic equations of quantum field theory, one can use Feynman diagrams and their associated "rules" to obtain the perturbative expansions that occur in the calculation of the transition probabilities. As an example, consider the mutual scattering of an electron $\left(e^{-}\right)$and a muon $\left(\mu^{-}\right)$. To determine the approximate probability of their scattering, we can first draw an inward directed line for the incoming electron, and one for the incoming muon (see Figure 1) and also two outward directed lines-one for the outgoing electron and one for the outgoing

3 Most of my presentation of how Feynman diagrams are usually used in today's theoretical particle physics is based on the textbook by Peskin and Schroeder (1995). 
muon. (For the purposes of this method, we think of the particles as moving along the horizontal axis; time is the vertical axis.)

To take into account the interaction of the particles, we use vertices in which an inward directed electron or muon line, an outward directed electron or muon line, and a wavy line representing a photon $(\gamma)$ meet, see Figure 2. (A photon is a quantum of the electromagnetic field.)

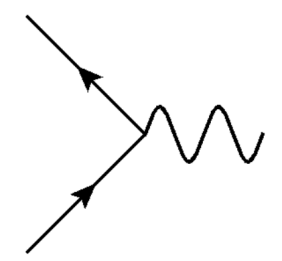

Figure 2. Adrian Wüthrich. Vertex to be inserted twice into the incomplete diagram of Figure 1.

The introduction of two such vertices suffices to represent the scattering of an electron and a muon off each other to a "first order" approximation in the perturbative expansion relative to the fine-structure constant $\alpha$. The result is the diagram in Figure 3, which can be read as showing the trajectory of an incoming electron and a muon, which emit or absorb a photon, before they fly out of the region where the interaction took place. Whether, or to what extent, such a reading is correct will be the topic of much of the following discussion.

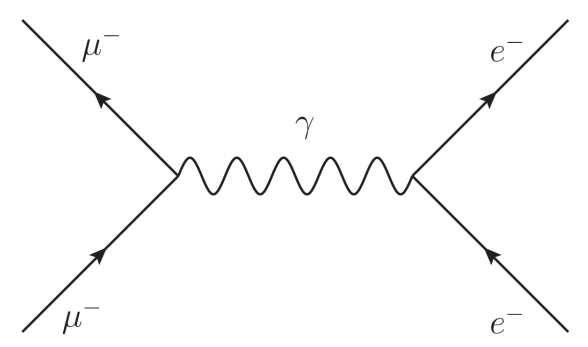

Figure 3. Adrian Wüthrich. Feynman diagram of "first order" (as explained in the text) used for the theoretical treatment of the mutual scattering of an electron $\left(\mathrm{e}^{-}\right)$and a muon $\left(\mu^{-}\right)$. The wavy line is associated with a photon ( $(\mathrm{\gamma})$, the quantum of the electromagnetic field.

The diagram can be translated into mathematical expressions according to the "Feynman rules," which associate each graphical element with a mathematical expression. ${ }^{4}$

\section{Drawing and interpreting Feynman diagrams}

In order to calculate the relevant observable quantities of a quantum field theoretical system, i.e. transition probabilities, it suffices to know the right

${ }^{4}$ For details see, e.g., Peskin and Schroeder (1995, 801-02). 
Feynman diagrams and the rules for translating them into the corresponding mathematical expressions. Of course, the obtained expressions still have to be evaluated quantitatively. In some highly idealized and restricted theories, such as the quantum theory of a single scalar field, this task may not be so difficult. However, for more comprehensive theories like quantum electrodynamics, or the field theory of the electroweak or strong nuclear interactions, the evaluation of these expressions often comprises an entire PhD thesis in theoretical physics.

In any case, for the calculation of the transition probabilities it does not matter through what kind of reasoning one arrived at the Feynman diagrams as long as they are the right ones. In particular, one can use the aforementioned physical considerations involving electrons, muons and their emission or absorption of a photon. These considerations, however, included several problematic statements such as those involving trajectories of particles in space and time. The usual notion of a trajectory is not compatible with the principles of a quantum theory; Heisenberg's uncertainty relation makes clear that we cannot simultaneously measure sharp values of the space and momentum coordinates of a particle, which is required for there to be a trajectory in the usual sense of the word.

Another difficulty that the aforementioned physical considerations have to face is that they imply the statement that there exists a photon in the intermediate state of the process. Yet, as I will discuss later in a bit more detail, the photon in the intermediate state is not always "on its mass-shell," i.e. its energy, momentum, and mass are not always related in the same way as with ideally observed, real photons. Therefore, the photon in the intermediate state cannot exist in the usual sense of the word; it is often said to exist "virtually" instead of really.

We therefore seem to be able to find the correct Feynman diagrams and, through their associated rules, the correct mathematical expressions by considerations which are clearly wrong. However, if physical considerations always lead us to the correct Feynman diagrams, we expect the considerations to contain at least some correct statements about the state of affairs in question. To most of us, I take it, it would seem highly unlikely, or even a "miracle," if the wrong considerations always led us to the correct Feynman diagrams and thus, ultimately, to true statements about the state of affairs in question.

In the following section I present a specific use of a Feynman diagram in the theoretical treatment of the scattering of electrons and muons. I will employ the concept of "models" to describe how Feynman diagrams and the physical considerations one uses in drawing them get at least something right about the processes one wants to describe and explain. 


\section{MOdels}

In the previous section I introduced the two principal objections to a physical interpretation of Feynman diagrams: they may erroneously suggest both that the usual concept of a particle trajectory is applicable to quantum theoretic objects, and that particles exist, in the usual sense of the word, in the intermediate states of a quantum theoretic transition process. Letitia Meynell (2008) proposes a similar classification of the objections against a physical interpretation of Feynman diagrams.

However, from the fact that Feynman diagrams are extensively used as calculation tools, and from the fact that some of their interpretations face serious difficulties, we cannot conclude that facilitating calculations is their only function, and that any type of interpretation is untenable. First of all, diagrams can simultaneously function as idealized representations of physical processes and as means by which one derives certain propositions about the processes in question. Second, the refutation of particular interpretations is not sufficient to rule out the possibility, in principle, of a coherent interpretation.

Let us examine an example in a completely different context to see how diagrams can serve as representations of a physical system and, at the same time, as a means to calculate characteristic quantities of the system. Consider, for instance, a schematic diagram of a massive body on an inclined plane (Figure $4)$.

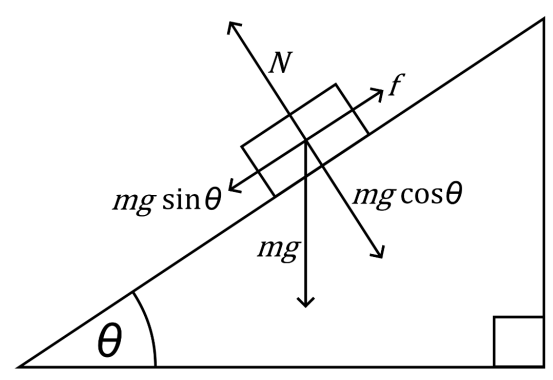

Figure 4. Schematic diagram of a massive body on an inclined plane. ${ }^{5}$

This diagram serves to reduce the real situation to the aspects that are relevant to the dynamics of the body, such as the forces that act on it. At the same time, the diagram serves to derive certain relations between the important quantities such as the relative length and angles of vectors, which represent the forces that act on the body. In a similar fashion, Feynman diagrams can be used to represent the relevant aspects of a physical situation and to calculate the interesting quantities.

5 "A free body diagram of a mass on an inclined plane" (2007) from en.wikipedia.org/ wiki/Inclined_plane. Creative Commons Attribution-Share Alike 3.0 Unported. 
For example, the Feynman diagram from Figure 3 represents the scattering of an electron and a muon, as being brought about by the emission and absorption of a photon by the muon or the electron. Although one must not infer from the diagram that the emission and absorption events (represented by the vertices of the diagram) take place at certain points in space-time, one can rightly infer from it that the change in the electron's or muon's momentum (which are the same by the principle of momentum conservation) is approximately equal to the momentum of the photon. This is because, in this rough representation which the diagram provides, the presence of a photon is the common cause for the changes in the other two particles' momenta. The photon mediates the interaction between the electron and the muon; it links or connects the two particles.

Along these lines, Feynman diagrams can be viewed as models of interactions of elementary particles. ${ }^{6}$ They allow us to explain certain aspects of the behavior of the system which they represent, even though we cannot take them to be accurate representations in every respect. For example, modeling the mutual scattering of an electron and a muon by the Feynman diagram from Figure 3 allow us to explain that, most of the time, the electron and the muon deflect each other only a little.

Without going into the details, the explanation works as follows. In the representation by the Feynman diagram, the interaction of the electron and the muon is brought about by the emission and absorption of a virtual photon. The lifetime of a virtual photon depends on how much it is "off the mass-shell." The virtual photon lives increasingly longer the nearer it is to its mass-shell. If and only if it lives longer, it can travel farther and thus mediate a force of longer range. Under normal circumstances, the longer the range of a force, the higher the rate of the reaction which is brought about by that force. Therefore, if and only if the virtual photon is near its mass-shell, the reaction rate is high.

On the other hand, according to the representation by the Feynman diagram of Figure 3, the energy and momentum of the virtual photon equal the changes in energy and momentum of the electron or the muon. A bit of calculation would show that, therefore, the virtual photon violates the mass-shell condition only a little if and only if the change in momentum of the electron and the muon are small, which means that they deflect each other only a little. Therefore, if and only if the particles deflect each other only a little, the photon is near its

${ }^{6}$ For notions of "models" which I think would be a good starting point to elaborate my account of the function and interpretation of Feynman diagrams, see R.N. Giere, Visual Models and Scientific Judgment, in Picturing Knowledge: Historical and Philosophical Problems Concerning the Use of Art in Science, ed. Brian Baigrie, (Toronto: University of Toronto Press, 1996, 296-302.); M. Suárez, An inferential conception of scientific representation, Philosophy of Science 71(5) (2004): 767-79; or G. Contessa, Scientific representation, interpretation, and surrogative reasoning, Philosophy of Science 74(1) (2007):, 48-68. 
mass-shell. Thus, together with the conclusion from the previous paragraph, we have shown that, if and only if the electron and the muon deflect each other only a little, the reaction rate is high (cf. Halzen and Martin 1984, 97).

In addition, the angular dependence of the reaction rate (or cross section) is entirely different-in fact, to first order in $\alpha$, there is none-when the photon line in the leading order diagram connects the vertex where the lines for the two incoming particles meet to the vertex where the lines for the two outgoing particles meet (see Figure 5). The interaction of an electron and a positron $\left(\mathrm{e}^{+}\right)$, which produces a final state containing a muon and an anti-muon $\left(\mu^{+}\right)$, is such a case. ${ }^{7}$ In the physicists' jargon the photon, in Figure 5, is said to be in the "s-channel" while in the case considered above (Figure 3) the photon was in the "t-channel" (Peskin and Schroeder 1995, 157). There, the photon line connected the two vertices in which the lines for the incoming and outgoing particles met.

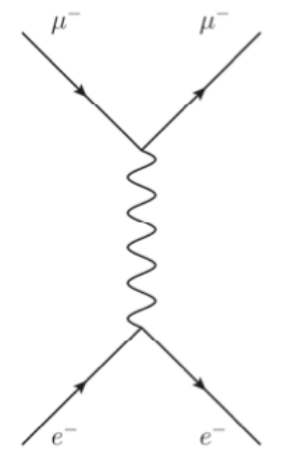

Figure 5. Adrian Wüthrich. Feynman diagram containing a photon line in the "s-channel" (as explained in the text).

In this way, the topology, as it were, of the Feynman diagrams captures an important aspect of the physical process. The distinct ways in which the photon line connects the lines for the incoming and outgoing particles corresponds to distinct angular dependencies of the cross section (in a rough approximation). This contrast to other Feynman diagrams contributes to the explanation based on the representation provided by the diagram of Figure 3.

In the interpretation I propose of the Feynman diagram of Figure 3, the contradictions to quantum mechanical principles are avoided because the interpretation leaves open many details-exactly those details, that is, which would contradict quantum mechanical principles. For instance, the interpretation does not claim anything about the exact time and place of the emission of the photon. But despite this lack of specificity, the more coarse-grained claims of the interpretation-for instance that the most relevant

7 Downward directed lines correspond to the oppositely charged partners of the particles which are represented by upward directed lines. 
common cause of the changes in momentum of the muon and the electron is the presence of a photon-may be correct.

Models are, in my opinion, an adequate conceptual framework to spell out this idea. A model is not a one-to-one representation of a real system; rather, it is an object (for instance a drawing or a three-dimensional material construct) which articulates only some of the relevant aspects of the real system. It would be wrong to interpret the representation by the model of the real system as claiming that the model and the real system are completely equal. ${ }^{8}$

Moreover, an interpretation of a model should not be rejected only because it seems difficult to develop in more detail. The reference to reality is not hampered by the fact that we, today, do not know how to add more details to the model. On the one hand, a model can be well justified on a coarse-grained level even if many details have to be left open. On the other hand, the eventual detailed elaboration of a model should not destroy the adequacy and reference to reality of the originally coarser model. We should, therefore, not reject a physical interpretation of Feynman diagrams-even though it is only partial-but think about how we can regard them as visual models.

\author{
ADRIAN WÜTHRICH \\ Institute for Philosophy \\ University of Bern \\ Sidlerstrasse 5 \\ 3012 Bern \\ Switzerland \\ awuethr@philo.unibe.ch
}

\title{
REFERENCES
}

Baigrie, Brian, ed. 1996. Picturing Knowledge: Historical and Philosophical Problems Concerning the Use of Art in Science. Toronto: University of Toronto Press.

Bohr, N., H. A. Kramers, and J. C. Slater. 1924. Über die Quantentheorie der Strahlung. Zeitschrift für Physik 24: 69-87.

Brown, James R. 1996. Illustration and inference. In Picturing Knowledge: Historical and Philosophical Problems Concerning the Use of Art in Science, ed. Brian Baigrie, 250-68. Toronto: University of Toronto Press.

Halzen, Francis, and Alan D. Martin. 1984. Quarks and Leptons: An Introductory Course in Modern Particle Physics. New York: John Wiley and Sons.

8 In fact, this is usually not even a goal of the modeler. If the model would be equal to the real system in (almost) every respect, it would be a copy of the real system rather than a model and would not bring to the fore the relevant or essential features of the system. 
A. Wüthrich

Interpreting Feynman Diagrams as Visual Models

Meynell, Letitia. 2008. Why Feynman diagrams represent. International Studies in the Philosophy of Science 22(1): 39-59.

Peskin, Michael E., and Daniel V. Schroeder. 1995. An Introduction to Quantum Field Theory. Boulder, CO: Westview Press.

Wüthrich, Adrian 2010. The Genesis of Feynman Diagrams. Dordrecht: Springer 